\title{
The difference between gender in terms of nomophobia in
} Turkey: a meta-analysis

\author{
Esin Avcı $\odot$ \\ Department of Statistics, Giresun University, Faculty of Arts and Sciences, Giresun, Turkey
}

\begin{abstract}
Objectives: Technology unfortunately even though making our life easier, causes a lot of psychological problems when it is not used reasonably. One of them is the inability to live without a smartphone: nomophobia. This meta-analysis study considered nomophobia in Turkey in terms of gender.

Methods: Using "nomophobia", "Nomophobia Scale ", "Nomophobia Questionnaire (NMP-Q)", "NMP-Q"and "smartphone" keywords, 9 electronic bibliographic databases from the internet were searched for studies related to the nomophobia scale. The inclusion criteria were determined as studies that the nomophobia questionnaire (NMP-Q) utilized to Turkish people, published in English or Turkish, and reported the questionnaire score by mean/standard deviation according to gender. The mean age and sample size ratio, which were thought to have an effect on heterogeneity, are analyzed by meta-regression.

Results: From the 9 electronic bibliographic databases, a total of 3370 studies were located, and only 10 meetings the inclusion criteria. It revealed that females are found to be more nomofobic than males according to Nomophobia Questionnaire (NMP-Q). The mean age and sample size ratio, which were thought to have an effect on heterogeneity, are analyzed by meta-regression.

Conclusions: The gender difference was found to be statistically non-significant in 2 of the 10 studies included in the meta-analysis. By enlarging the sample size, which is one of the advantages of meta-analysis, the difference between gender is determined more accurately.

Keywords: Nomophobia questionnaire, NMP-Q, meta-analysis, gender, smartphone
\end{abstract}

W ith the latest developments, mobile phones are no longer used only for communication with another person via verbal communication or text message, but also provide versatile communication possibilities with smartphone features. Smartphones have become attractive to millions of people because of advanced capacity, constantly updated operating systems, and outperforming mobile phones in terms of processing power [1]. According to the "TURKSTAT Household Information Technology Usage Survey (2004-2018)", the rate of having mobile phones/smart- phones in households is increased from 53.7 percent to 98.7 between 2004 to 2018 . While the use of mobile phones and smartphones facilitates our lives in all areas, some negative effects such as addiction or anxiety occur.

Nomophobia, which is called the new phobia of the modern age, comes from the English word nomophobia "NO MObile PHOBIA". In psychology, it is defined as the irrational fear experienced by an individual when he / she cannot access or communicate on their mobile device $[2,3]$. 
In nomophobia, the individual begins to feel anxious when he forgets to take his phone with him, when his phone is out of charge or out of range. This anxiety negatively affects the concentration of the individual on daily work in his life [4].

In order to determine the nomophobia level Nomophobia Questionnaire (NMP-Q) is developed by Yıldırım and Correira [3]. The Turkish version is formatted by Y1ldirım et al. [5]. It contains a 7-point Likert type, 20 items, and four subdimensions. The validity and reliability of the scale were assessed and referred to it. Four subdimensions are named as follows; unable to access information (1-4 items), giving up convenience (5-9 items), unable to communicate (10-15 items), and losing connectedness (16-20 items). By summing up each item response score, the nomophobia scale scoring is ranged between 20 and 140 . According to mild, moderate, and severe nomophobia levels, the scoring is ranged 21-59, 60-99, and 100140, respectively [3].

After Yildirım et al. [5] transform the Nomophobia Questionnaire (NMP-Q) to the Turkish version, the application of the transformed questionnaire in Turkey has increased. Numerous studies have been independently conducted across Turkey to reveal nomophobia. Yildirim et al. [5] showed that the rate of the nomophobic behavior of university students is $42.6 \%$. Onal and Onal [6] aimed to determine high school students' use of smartphones and their nomophobia levels, for this purpose 767 students participated in the research. The results showed that high school students were nomophobic at a moderate level [6]. Adnan and Gezgin [7] utilized the transformed scale on 433 university students found the level of nomophobia is over average. Hosgor and Hosgor [8] conducted on the university students in Istanbul who studying on Department of Health Management, they revealed also over average nomophobic behavior. Güllüce et al. [9] searched the relationship between subjective well-being and nomophobia levels of Ardahan University students. They determined that men are less nomophobic than women [9]. Gezgin and Cakir [10] analyzed the prevalence of nomophobia among high school students according to smartphone usage, gender, class levels, mother's and mother's education level, smartphone usage time, and mobile internet usage. Yavuz et al. [11] examined nomophobia on an adolescent population related to alexithymia and metacognitive prob- lems. Arslan et al. [12] conducted research on teachers to find out the correlation of nomophobia and fear of missing out. Akhoroz [13] examined the correlation between the nomophobia and personality traits among preservice teachers. Arpaci et al. [14] related the nomophobia to attachment for college students and concluded the gender factor is important in mindfulness-based treatments. Çelik İnce [15] searched the correlation between self-esteem and obesity and nomophobia. The analysis results demonstrated moderate nomophobia and non-significant correlation [15].

In parallel with the increase in the generality of smartphone using, nomophobia, and the related scientific studies number are also increasing. Hence, it is appropriate to use meta-analysis, which is a statistical method combining and interpreting the results of more than one scientific study. This meta-analysis examined all studies published between January 2016 and December 2020.

The main purpose of this study is to determine whether nomophobia differs according to gender by meta-analysis. On the other hand, to assess the effects of the mean age of each study and sample size ratio on the difference mean, meta-regression is applied. The analyzes are made using the "meta" and "metafor" packages in the $\mathrm{R}$ package.

\section{METHODS}

In recent years meta-analysis has gained importance with the ease of access to scientific studies on any subject. Meta-analysis is a statistical analysis method that allows combining the results obtained from many studies on a specific subject and independent from each other [16]. Since meta-analysis is considered as the combination of the studies, it is important to determine all publications related to the subject.

Meta-analysis can be applied in combining experimental studies rather than theoretical studies. The meta-analysis method differs according to the reported summary statistics.

The benefits of the meta-analysis are expressed as follows: by increasing the sample size making a decision about the uncertainty and estimating the effect size in case of conflict of independent study results [17]. 
Effect size is the basic unit of meta-analysis and represents the direction and size of the relationship of interest. By standardizing the results of the studies, it provides the opportunity to directly compare. Mean difference, correlation coefficient and odds ratio are examples of different types of effect sizes $[16,18]$. The calculation of effect size differs according to study purpose, design and data type. If the data type nominal, continuous or indicate a relationship respectively the proportions, mean, and the correlation coefficient are used [19]. It is possible to convert effect sizes to each other by using transformation formulas [16].

Fixed and random effect models are the two main models used in meta-analysis. The characteristic structure of the study and the source of error are important in model selection. When studies are obtained from published literature, it is more appropriate to use the random effect model [16].

One of the graphics that provides a better understanding of the results visually in meta-analysis is the forest graph. This graph shows the effect size of each study, $95 \%$ or $99 \%$ confidence intervals, weights, and combined effect size. This graph provides information about the variability among the estimates of each study [20].

Mean and variance (or standard deviation) are the main descriptive statistics in the meta-analysis of continuous data. Usually, to compare the means of two independent groups the raw mean or the standardized mean difference are preferred effect sizes. The raw (Unstandardized) mean difference is given in Eq. (1) which is used when all studies in the analysis use the same scale (for example blood pressure). Standardized mean differences (d) and (g) are given in Eq. (2) and Eq. (7) used when the different studies use different instruments (such as different psychological or educational tests) to assess the outcome. Since the scale of measurement will differ from study to study so it will not meaningful to combine raw mean difference.

The raw (Unstandardized) mean difference and standardized mean difference for sample estimate are given as;

$$
\begin{aligned}
& D=\bar{X}_{1}-\bar{X}_{2} \\
& d=\frac{\bar{X}_{1}-\bar{X}_{2}}{S_{\text {within }}}
\end{aligned}
$$

The variance of $\mathrm{D}$ and $\mathrm{d}$ are given as;

$$
\begin{aligned}
& V_{D}=\frac{S_{1}^{2}}{n_{1}}+\frac{S_{2}^{2}}{n_{2}} \\
& S_{\text {within }}=\sqrt{\frac{\left(n_{1}-1\right) S_{1}^{2}+\left(n_{2}-1\right) S_{2}^{2}}{n_{1}+n_{2}-2}} \\
& V_{d}=\frac{n_{1}+n_{2}}{n_{1} n_{2}}+\frac{d^{2}}{2\left(n_{1}+n_{2}\right)}
\end{aligned}
$$

where $\bar{X}_{1}, \bar{X}_{2}, S_{1}^{2}$ and $S_{2}^{2}$ be the sample mean and variance of the two groups, and $n_{1}$ and $n_{2}$ be the sample size in the two groups. In small samples, a correction factor is used to remove a slight bias of $d$ that called Hedges'g. $\bar{X}_{1}$

Differences in study design (scale, population,

$$
\begin{aligned}
& J=1-\frac{3}{4 s d-1} \\
& g=J \times d \\
& V_{g}=J^{2} \times V_{d}
\end{aligned}
$$

etc.) lead to heterogeneity. Therefore, heterogeneity analysis is important in the meta-analysis, and heterogeneity between studies can be tested with various statistical tests. The heterogeneity concern the true variance, not sampling error. Commonly used measurements in decomposing true variance; $\mathrm{Q}$ is the statistic (squared of weighted deviations) and the ratio of the true heterogeneity in the total observed variance $\left(\mathrm{I}^{2}\right)$. The $\mathrm{I}^{2}$ statistic takes values between $0 \%$ and $100 \%$, regardless of the effect size type (mean, ratio or correlation) used in the meta-analysis, values at $25 \%, 50 \%$ and $75 \%$ are considered low, medium and high heterogeneity, respectively.

In revealing the causes of heterogeneity on the basis of moderator variable (covariate or covariant); subgroup analysis is used for the categorical moderator variable, and multiple meta-regression analysis is used for the continuous moderator variable [16]. In this study, briefly mentioned on meta-regression.

\section{Meta-regression}

In general, regression is defined as a model that determines the relationship between a dependent variable and an independent variable (s). For given $n$ observations the model for regression is given as; 
Where $y_{i}(i=1,2, \ldots, n)$ is a observed values, $\beta k \times 1$ coefficients vector, $x_{i}$ is a $1 \times k$ vector of covariate.

$$
y_{i}=x_{i}^{\prime} \beta+\varepsilon_{i} \quad \varepsilon_{i} \sim N\left(0, \sigma_{i}^{2}\right)
$$

While classic regression based on individual observations, the meta-regression based on study-level summary data.

For given $\mathrm{n}$ study fixed-effects meta-regression is given in $\mathrm{Eq}(10)$,

Even though fixed-effects assume zero heterogeneity, it used mostly in replicated experiment appli-

$$
\begin{array}{cr}
y_{i}=x_{i}^{\prime} \beta+\varepsilon_{i} & y_{i} \sim N\left(\theta_{i}, \sigma_{i}^{2}\right), \\
\theta_{i} \sim N\left(\theta+x_{i}^{\prime} \beta, \sigma_{a}^{2}\right) & \varepsilon_{i} \sim N\left(0, \sigma_{i}^{2}\right)
\end{array}
$$

cations [16].

For given $\mathrm{n}$ study random-effects meta-regression is given in $\mathrm{Eq}(11)$,

$$
\begin{aligned}
& y_{i}=x_{i}^{\prime} \beta+u_{i}+\varepsilon_{i} \quad y_{i} \sim N\left(\theta_{i}, \sigma_{a}^{2}+\sigma_{i}^{2}\right), \\
& \theta_{i} \sim N\left(\theta+x_{i}^{\prime} \beta, \sigma_{a}^{2}\right), \\
& u_{i} \sim N\left(0, \tau^{2}\right), \\
& \varepsilon_{i} \sim N\left(0, \sigma_{i}^{2}\right)
\end{aligned}
$$

Where $y_{i}(i=1,2, n)$ is an observed values, $\beta k \times$ 1 coe fficients vector, $x_{i}$ is a $1 \times k$ vector of covariate values in study i [21].

The significance of the regression coefficient $\left(H_{0}: \beta_{i}=0\right)$ can be evaluated with the $\mathrm{Z}$ test;

$$
Z=\frac{\hat{\beta}_{j}}{S e_{\widehat{\beta}_{j}}}
$$

where $\hat{\beta}_{j}$ is the least square estimator of the parameter and the $S e_{\widehat{\beta}_{j}}$ is the estimated standard error of $\hat{\beta}_{j}$. under $H_{0}$ Eq. (12) has the standard normal distribution.

A bubble plot is a useful tool that plotting the effect size versus a continuous covariate. The fitted line with the circle demonstrates the estimates from each study, and the circle size differing with the weighted of studies.

\section{RESULTS}

This study search whether nomophobia, which is the phobia of the inability to live without a smart- phone, that occurs with the widespread use of smartphones, differs according to gender. Using "nomophobia", "Nomophobia Scale", "Nomophobia Questionnaire (NMP-Q)", "NMP-Q"and "smartphone" keywords, 9 electronic bibliographic databases searched the studies related to the nomophobia scale. The inclusion criteria were studies that the nomophobia questionnaire (NMP-Q) utilized to Turkish people, published in English or Turkish, and report the questionnaire score by mean/standard deviation according to gender. A total of 3370 studies were located, with 10 meeting the inclusion criteria (Fig. 1).

Some of the studies are reported as a mean of Likert scale scores and others as a total score. To uniform, these reported studies, by multiplying 20 (number of items in the scale) to mean of Likert scale scores the total score is obtained [3].

Based on gender difference the information of included 10 studies in the meta-analysis is given in Table 1.

In order to determine the difference between gender according to the mean nomophobia scale score, a meta-analysis conducted using standard mean difference effect size (SMD). The results summarized in Table 2.

It can be seen from Table 2 that the mean nomophobia scale score for both the fixed and random effect model varies according to gender $(p<0.05)$. For both models, the mean score of females was found to be higher than males. Also, heterogeneity is statistically significant $(p<0.05)$. The cause of heterogeneity might be due to utilizing different population characteristics (age, city, etc.).

Since the included studies in the meta-analysis are compiled from published literature the use of the random-effect model is considered more appropriate. Therefore, the forest plot only shows the random-effect model results.

The Forest plot divided into eleven columns (Fig. 2 ). The results of each study results are displayed in rows. The first column ("study") lists the identification of each study included in the meta-analysis. The second and fifth columns represents the total number of participants of each study. The third and sixth columns displays the male and female mean values of NMP-Q scale of each study. The fourth and seventh columns displays the male and female standard deviation values of NMP-Q scale of each study. The eighth column rep- 


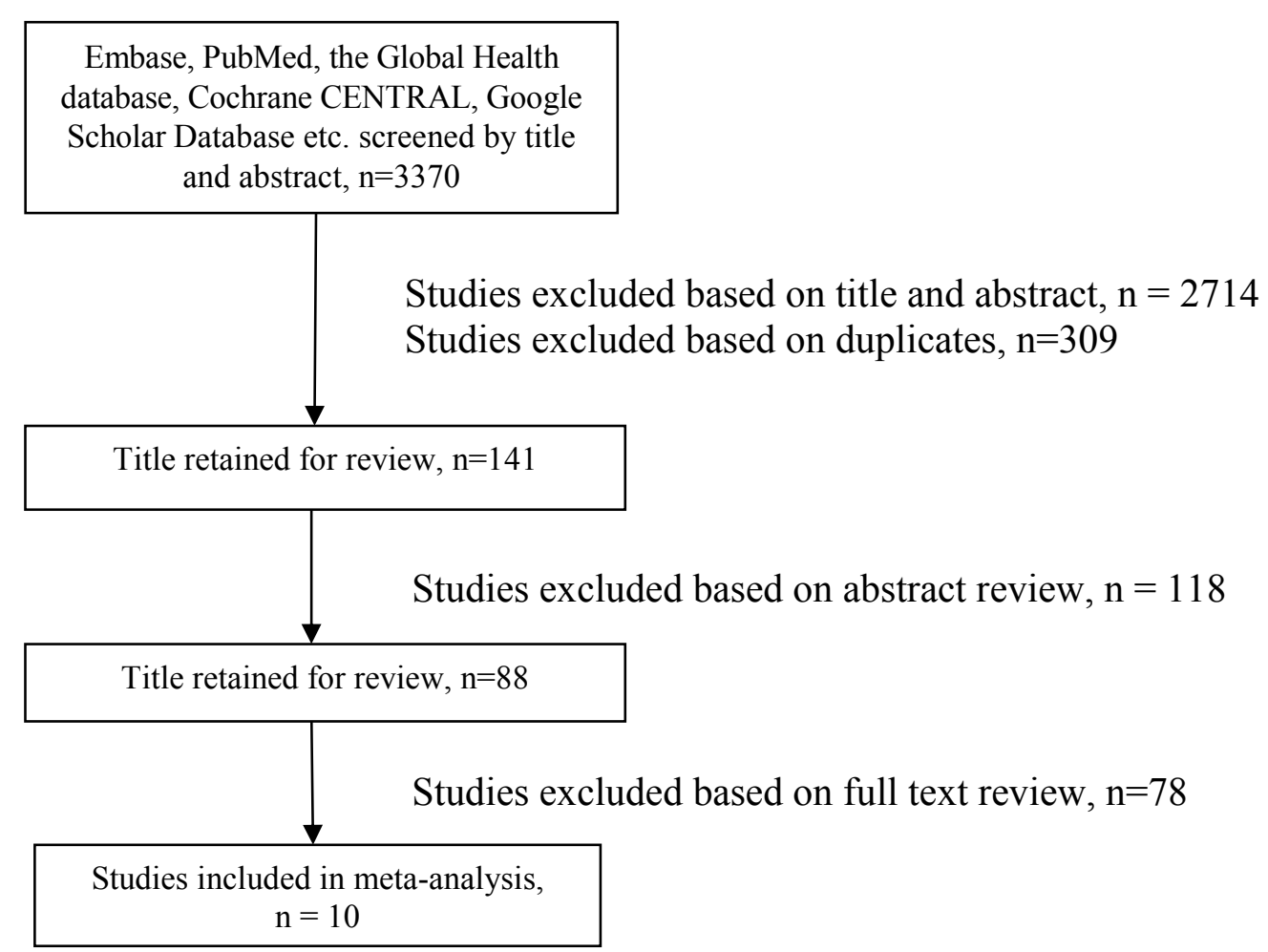

Fig. 1. Summary of literature search and selection of studies.

resents the standardized mean difference (effect estimates) of each study included in the meta-analysis and related confidence interval. The effect estimate of each study depicted with a box situated in line. The size of the box is directly related to the reciprocal of the variance (weighting) of each study in the meta-analysis. The length of the confidence interval (CI) represented with the horizontal line (Whiskers) through the box. In the case of the long lines, the confidence interval gets wider and this means that the less precise the study results. The ninth column represents the effect estimates of each study numerically. The tenth column represents the confidence interval of each study numerically. The last column depicts the weight (in \%) indicates the weighting or influence of each study on the overall results of the meta-analysis of all included studies.

In the last row of the graph, the diamond illustrates

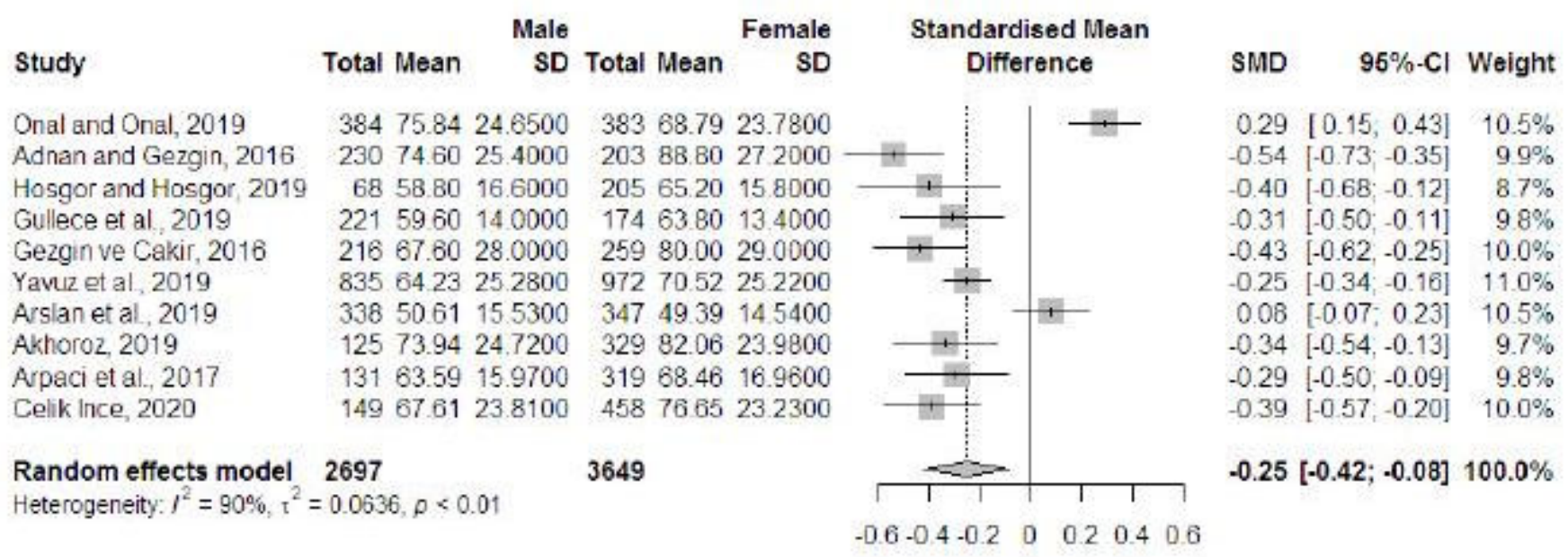

Fig. 2. Forest plot of standardized mean difference. 


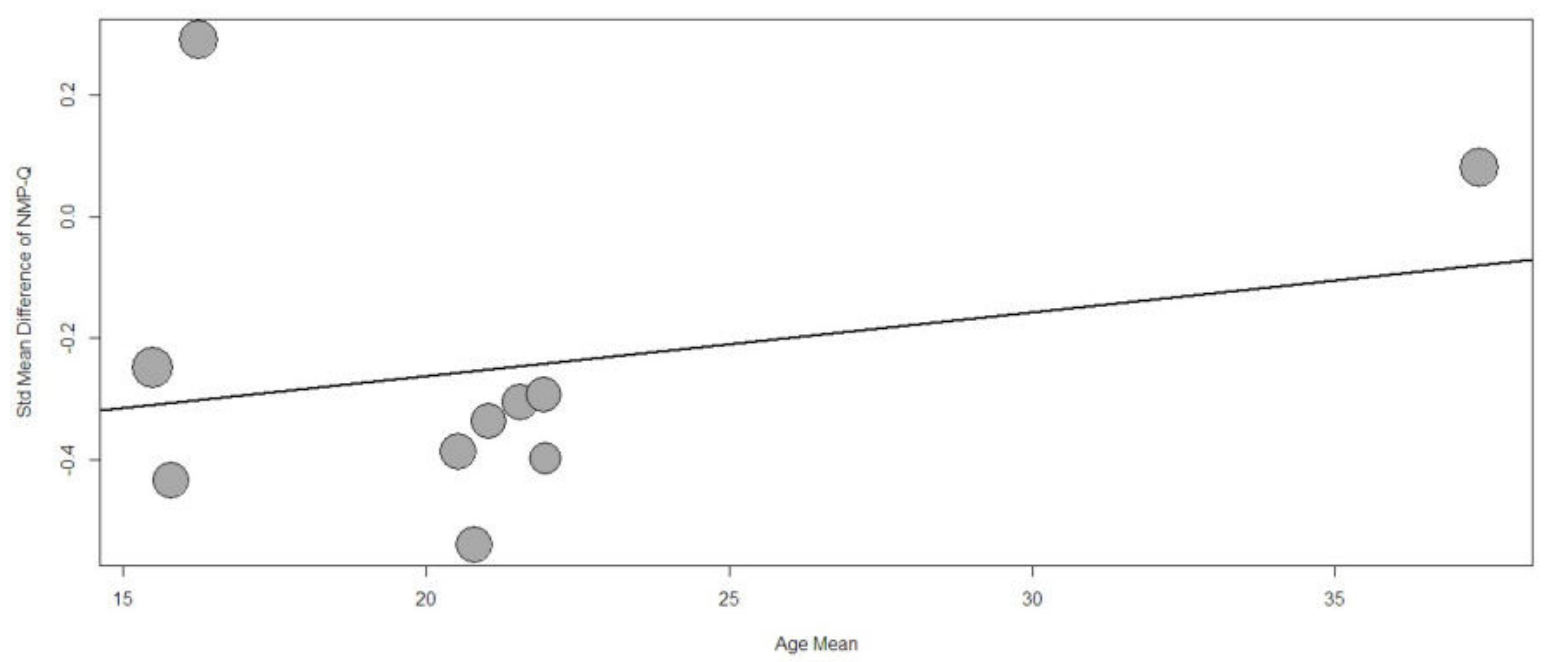

Fig. 3. Bubble plot of age mean.

the overall result of the meta-analysis. The width of the diamond indicates the width of the overall confidence interval. The most reliable test for heterogeneity is given at the bottom of the graph on the left hand $\left(\mathrm{I}^{2}\right)$. Checking the overlap of the confidence interval is a useful visual guide to assessing heterogeneity. If the confidence interval of all studies overlaps than studies are regarded as homogeneous.

The Forest plot shows that, the standardized mean difference for gender ranging between 0.08 and -0.54 . Eight out of ten studies received negative values and the overall standardized mean difference is -0.25 . This shows that females have a higher mean nomophobia score than males. The confidence intervals of studies are narrow so the results of the studies are precision. On the other hand, whether the confidence intervals contain zero or not makes it easy to determine whether there is a statistical difference between the groups. While only one out of ten studies showed no difference in mean nomophobia score for females and males [12], this difference appears to be significant for the overall effect. The weighting of each study included in the meta-analysis changed between 8 and 11. The study with the smallest variance influenced the most to the overall effect result [11]). This can be seen from the box and the horizontal line (Whiskers) through the box. Yavuz et al.'s study [11] give relatively the biggest box and narrowest line. The heterogeneity can

Table 1. Information of included studies in the meta-analysis

\begin{tabular}{|c|c|c|c|c|c|c|}
\hline \multirow[t]{2}{*}{ Study } & \multicolumn{2}{|c|}{ Male } & \multicolumn{2}{|c|}{ Female } & \multirow{2}{*}{$\begin{array}{c}\text { Mean } \\
\text { Age }\end{array}$} & \multirow{2}{*}{$\begin{array}{c}\text { Ratio of Sample } \\
\text { Size of Males to } \\
\text { Females }\end{array}$} \\
\hline & $\begin{array}{l}\text { Sample } \\
\text { size }\end{array}$ & Mean \pm SD & $\begin{array}{l}\text { Sample } \\
\text { size }\end{array}$ & Mean \pm SD & & \\
\hline Onal and Onal, 2019 [6] & 384 & $75.84 \pm 24.65$ & 383 & $68.79 \pm 23.78$ & 16.25 & 1.00 \\
\hline Adnan and Gezgin, 2016 [7] & 230 & $74.6 \pm 25.4$ & 203 & $88.8 \pm 27.2$ & 20.79 & 1.13 \\
\hline Hosgor and Hosgor, 2019 [8] & 68 & $58.8 \pm 16.6$ & 205 & $65.2 \pm 15.8$ & 21.97 & 0.33 \\
\hline Güllüce et al., 2019 [9] & 221 & $59.6 \pm 14$ & 174 & $63.8 \pm 13.4$ & 21.55 & 1.27 \\
\hline Gezgin and Cakir, 2016 [10] & 216 & $67.6 \pm 28$ & 259 & $80 \pm 29$ & 15.8 & 0.83 \\
\hline Yavuz et al., 2019 [11] & 835 & $64.23 \pm 25.28$ & 972 & $70.52 \pm 25.22$ & 15.5 & 0.86 \\
\hline Arslan et al., 2019 [12] & 338 & $50.61 \pm 15.53$ & 347 & $49.39 \pm 14.54$ & 37.38 & 0.97 \\
\hline Akhoroz, 2019 [13] & 125 & $73.94 \pm 24.72$ & 329 & $82.06 \pm 23.98$ & 21.02 & 0.38 \\
\hline Arpaci et al., 2017 [14] & 131 & $63.59 \pm 15.97$ & 319 & $68.46 \pm 16.96$ & 21.94 & 0.41 \\
\hline Çelik İnce, 2021 [15] & 149 & $67.61 \pm 23.81$ & 458 & $76.65 \pm 23.23$ & 20.53 & 0.33 \\
\hline
\end{tabular}

$\mathrm{SD}=$ standard deviation 


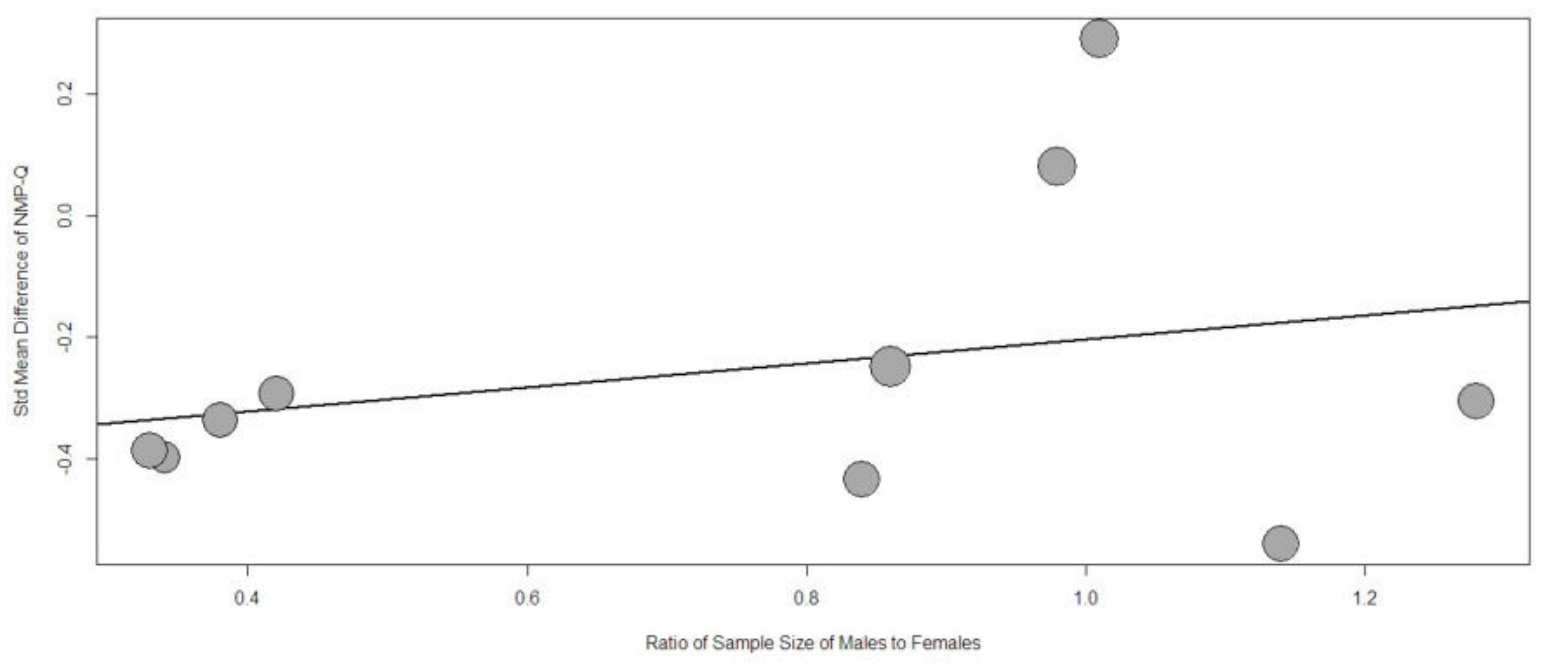

Fig. 4. Bubble plot of ratio of sample size.

be observed by the not overlapping confidence interval of all studies (Fig. 2).

Finally, the overall standardized mean difference -0.25 (95\% CI, -0.42 to -0.08$)$, there is a statistically significant difference between females and males, meaning that the mean nomophobia score is higher in the females compared to the males. Also indicated significant between-study heterogeneity $(\mathrm{Q}=87.66, p<$ $0.05)$ with $I^{2}=90 \%$, which means that $90 \%$ of observed variance comes from real differences between studies and, as such, can potentially be explained by study-level covariates.

Although the literature is searched for the same scale, it is examined by meta-regression whether the age difference of the population to which the scale is utilized is effective in heterogeneity. The mean age ranged between 15.5 and 37.38 (Table 1).

Table 3 shows that the regression coefficients are not statistically significant $(p>0.05)$. Therefore, it is concluded that the mean age covariate does not affect the mean nomophobia scale score in terms of gender. This conclusion can also be reached according to whether the $95 \%$ confidence interval of each coefficient includes zero or not. The $95 \%$ confidence interval of both coefficients contains zero.

The linear model obtained by meta-regression is graphically displayed with a bubble graph. It is given in Fig. 3 for age mean. The circles in the graph are proportional to the weight of each study. The ten studies considered seem to have almost the same weight. The middle line shows the estimated values of each study. Although it is not statistically significant, it appears from the graph that there is difference in terms of mean nomophobia scale score between males and females as the age increases. In terms of gender, the mean nomophobia scale score difference is mostly in favor of males between the ages of 15-20, this difference changing in favor of females the ages of $21-25$,

Table 2. Results of meta-analysis

\begin{tabular}{lccccc}
\hline Model & SMD & $\mathbf{9 5 \%}$ CI & $\mathbf{z}$ & $\boldsymbol{p}$ value & Test of heterogeneity \\
\hline Fixed effect & -0.20 & {$[-0.25-0.15]$} & -7.76 & $0.0001^{*}$ & $\begin{array}{c}\mathrm{Q}=87.88 \\
(p \text { value }=0.0001)\end{array}$ \\
Random effects & -0.25 & {$[-0.42-0.08]$} & -2.93 & $0.0034^{*}$ & \\
\hline
\end{tabular}

Table 3. Results of meta-regression analysis for age mean

\begin{tabular}{lccccc}
\hline & Coefficient estimate & Coefficient SE & $\mathbf{z}$ & $\boldsymbol{p}$ value & $\mathbf{9 5 \%}$ CI \\
\hline Intercept & -0.47 & 0.33 & -1.43 & 0.15 & {$[-1.12-0.17]$} \\
Age mean & 0.01 & 0.01 & 0.70 & 0.48 & {$[-0.02-0.04]$} \\
\hline
\end{tabular}


Table 4. Results of meta-regression analysis for the ratio of sample size

\begin{tabular}{lccccc}
\hline & Coefficient estimate & Coefficient SE & $\mathbf{z}$ & $\boldsymbol{p}$ value & $\mathbf{9 5 \%}$ CI \\
\hline Intercept & -0.40 & 0.21 & -1.91 & 0.06 & {$[-0.81-0.01]$} \\
Ratio of sample size & 0.20 & 0.25 & 0.79 & 0.43 & {$[-0.29-0.69]$} \\
\hline
\end{tabular}

for over 35 ages this difference again changes in favor of males; the score of males increases more than females.

In four of the ten studies included in the metaanalysis, the sample sizes of males and females are different. To determine whether this difference is important for heterogeneity, the ratio of a males sample size to a females sample size is examined by meta-regression. The ratio is ranged between 0.33 and 1.28 (Table 1).

Table 4 indicates that the regression coefficients are not statistically significant ( $\mathrm{p}>0.05)$. Therefore, it is concluded that the ratio of sample size covariate does not affect the mean nomophobia scale score in terms of gender. This conclusion can also be reached according to whether the $95 \%$ confidence interval of each coefficient includes zero or not. The $95 \%$ confidence interval of both coefficients contains zero.

Ones again the linear model obtained by meta-regression is graphically displayed with a bubble graph. It is given in Fig. 4 for the ratio of sample size.

The circles in the graph are proportional to the weight of each study. The ten studies considered seem to have almost the same weight. The middle line shows the estimated values of each study. Although not statistically significant, interesting results are obtained from the bubble graph. It can be seen from the graph that females have higher than males mean nomophobia scale scores in case of the ratio of sample size is smaller or greater than 1 . However, when this ratio is 1 or very close to 1 the mean score of males is higher than females (Fig. 4).

\section{DISCUSSION}

In the age of information, the features of smartphones are increasing day by day depending on the need. Especially with the pandemic we have been experiencing since 2020, phones are not only communication and messaging tools, but meeting, education, banking, food ordering, food shopping, socializing, etc. every need is at our fingertips. Unfortunately, a device that meets such needs becomes indispensable.

In this study, the difference between gender was investigated based on the Nomophobia Questionnaire (NMP-Q). Adnan and Gezgin [7] utilized the transformed scale on 433 university students' nomophobia was observed to make no difference for gender. The difference between gender was statistically significant for 273 students who were studying at the Department of Health Management [8]. Again, the difference by gender was found to be statistically significant in the scale applied on 395 university students [9]. A similar result was obtained for 450 university students [14]. In the case of the study conducted on 607 nursing students, the difference between gender was significant also [15].

When the study group consisted of 475 high school students, the difference was statistically significant for gender [10]. Also, when the study was conducted on 1817 participants $(n=972,54 \%$ female, $n=835,46 \%$ male) the difference between gender was statistically significant [11]. For 765 adolescents participants, the difference was statistically significant for gender [6].

To examine teachers' nomophobia, data were collected from a total of 685 teachers, no difference was observed between gender [12]. But for preservice teachers, the difference was statistically significant [13].

Except for the teachers' study group, the nomophobia scores of females were higher than males were observed. The gender difference was found statistically non-significant in 2 of 10 studies included in the metaanalysis.

\section{CONCLUSION}

It is no longer just for calling and messaging, but for banking, entertainment, etc. smartphones, which are also used for, have become a part of our lives. Un- 
fortunately, misusing this technological convenience leads to addiction in some people. The inability to live without a smartphone is called nomophobia (NO MObile PHOBIA) in psychology. The Nomophobia Questionnaire (NMP-Q) has been developed to measure the level of nomophobia.

With the increasing scientific studies on this subject, the necessity to combine and interpret these studies statistically is revealed. In this study, the difference in mean nomophobia scale score according to gender is discussed by meta-analysis. 10 studies are included in the analysis according to the inclusion criteria. As a result of the analysis, it is determined that females had higher mean nomophobia scale scores than males.

The mean age and sample size ratio, which were thought to have an effect on heterogeneity, are analyzed by meta-regression. Although the effect of the two covariates considered is not statistically significant in heterogeneity, females between the ages of 2025 have higher mean scores than males, while this situation reverses at the other ages. An interesting result is obtained for the sample size ratio. When the ratio is 1 or very close to 1 , males have a higher mean score than females, while the ratio is less or more than 1 , the mean score of females has increased than males. This result may be totally coincidental. For making more accurate inferences, more studies must be utilized.

Most of the studies discussed in this study were applied to student groups. Applying nomophobia studies to different sample groups is important in determining the difference between gender in the population.

\section{Authors' Contribution}

Study Conception: EA; Study Design: EA; Supervision: EA; Funding: EA; Materials: EA; Data Collection and/or Processing: EA; Statistical Analysis and/or Data Interpretation: EA; Literature Review: EA; Manuscript Preparation: EA and Critical Review: EA.

\section{Conflict of interest}

The authors disclosed no conflict of interest during the preparation or publication of this manuscript.

\section{Financing}

The authors disclosed that they did not receive any grant during conduction or writing of this study.

\section{REFERENCES}

1. Bian M, Leung L. Linking loneliness, shyness, smartphone addiction symptoms, and patterns of smartphone use to social capital. Soc Sci Comput Rev 2015;33:61-79.

2. King ALS, Valença AM, Silva AC, Baczynski T, Carvalho MR, Nardi AE. Nomophobia: dependency on virtual environments or social phobia? Comput Human Behav 2013;29:140-4.

3. Yildirim C, Correia AP. Exploring the dimensions of nomophobia: development and validation of a self-reported questionnaire. Comput Human Behav 2015;49:130-7.

4. Dixit S, Shukla H, Bhagwat A, Bindal A, Goyal A, Zaidi A, et al. A study to evaluate mobile phone dependence among students of a medical college and associated hospital of central India. Indian J Community Med 2010;35:339-41.

5. Yildirim C, Sumuer E, Adnan M, Yildirim S. A growing fear: prevalence of nomophobia among Turkish college students. Information Development 2016;32:1322-31.

6. Onal N, Onal TN. Nomophobia: I can not live without my smartphone! Croatian J Educ 2019;22:487-513.

7. Adnan M, Gezgin DM. [A modern phobia: prevalence of nomophobia among college students]. Ankara University, Journal of Faculty of Educational Sciences 2016;49:141-158. [Article in Turkish]

8. Hosgor H, Hosgor GD. The relationship among nomophobia, fear of missing out and demographic variables: example of the healthcare manager candidates. Current Addiction Research 2019;3:16-24.

9. Güllüce AC, Kaygin E, Borekci NE. [Determination of the relationshıp between the level of nomophobia and subjective wellbeing of university students: Ardahan case]. Hacettepe University Journal of Economics and Administrative Sciences 2019;37:65174. [Article in Turkish]

10. Gezgin DM, Çakır O. Analysis of nomofobic behaviors of adolescents regarding various factors. Int $\mathrm{J}$ Human Sci 2016;13:2504-19.

11. Yavuz M, Altan B, Bayrak B, Gunduz M, Bolat N. The relationships between nomophobia, alexithymia and metacognitive problems in an adolescent population. Turk J Pediatr 2019;61:345-51.

12. Arslan H, Tozkoparan SB, Kurt AA. [Examination of Nomophobia and Fear of Missing Out Among Teachers]. Erzincan Üniversitesi Eğitim Fakültesi Dergisi 2019;21:237-56. [Article in Turkish]

13. Akhoroz M. [Examining the relationship between personality traits and nomophobia among preservice teachers]. Unpublished master's thesis. Middle East Technical University, Ankara. 2019. [Article in Turkish]

14. Arpaci I, Baloglu M, Ozteke Kozan H, Kesici S. Individual differences in the relationship between attachment and nomophobia among college students: the mediating role of mindfulness. J Med Internet Res 2017;19:e404.

15. Çelik İnce S. Relationship between nomophobia of nursing students and their obesity and self-esteem. Perspect Psychiatr Care 2021;57:753-60.

16. Borenstein M, Hedges LV, Higgins JPT, Rothstein HR. Introduction to Meta-Analysis. Chichester: Wiley, UK. 2009. 
17. Sacks HS, Berrier J, Reitman D, Ancona-Berk VA, Chalmers TC. Meta-analyses of randomized controlled trails. N Eng J Med 1987;316:450-5.

18. Littel HJ, Corcoran J, Pillai V. Systematic Reviews and MetaAnalysis. New York: Oxford University Press. 2008.

19. Cohen LM, Manion L, Morrison K. Research Methods in Ed- ucation. London: Routledge. 2007.

20. Sutton AJ, Abrams KR, Jones DR, Sheldon TA, Song F. Methods for Meta-Analysis in Medical Research. Tronto: John Wiley \& Sons. 2000.

21. Harbord RM, Higgins JPT. Meta-regression in Stata. Stata J 2008;8:493-519. 